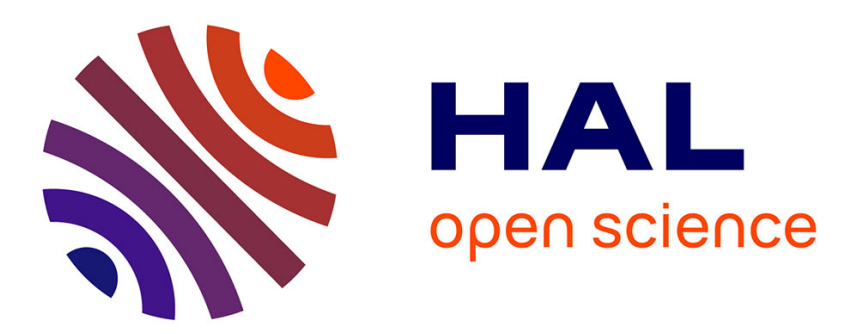

\title{
Quand la valeur des déterminants épithètes dépend de leur position dans le syntagme: le cas des adjectifs indéfinis en russe contemporain
}

Christine Bonnot

\section{To cite this version:}

Christine Bonnot. Quand la valeur des déterminants épithètes dépend de leur position dans le syntagme: le cas des adjectifs indéfinis en russe contemporain. 2011. halshs-00675372

\author{
HAL Id: halshs-00675372 \\ https://shs.hal.science/halshs-00675372 \\ Preprint submitted on 29 Feb 2012
}

HAL is a multi-disciplinary open access archive for the deposit and dissemination of scientific research documents, whether they are published or not. The documents may come from teaching and research institutions in France or abroad, or from public or private research centers.
L'archive ouverte pluridisciplinaire HAL, est destinée au dépôt et à la diffusion de documents scientifiques de niveau recherche, publiés ou non, émanant des établissements d'enseignement et de recherche français ou étrangers, des laboratoires publics ou privés. 


\section{Quand la valeur des déterminants épithètes dépend de leur position dans le syntagme : le cas des adjectifs indéfinis en russe contemporain}

Christine BONNOT ${ }^{1}$

\section{Objet de l'étude et hypothèse générale}

Bien que le russe soit une langue sans articles, il possède une grande variété d'adjectifs indéfinis dont l'emploi peut parfois s'avérer obligatoire. Qu'ils construisent une référence spécifique ou non spécifique, ces adjectifs ont pour fonction commune d'indiquer de quelle façon une occurrence ${ }^{2}$ de la notion désignée par le substantif est sélectionnée au sein de sa classe notionnelle. Ce faisant, ils l'individualisent en soulignant qu'elle possède des propriétés qui, bien que non explicitées ou non explicitables, la distinguent des autres occurrences incarnant la même notion. C'est pourquoi leur emploi est en principe exclu avec des substantifs renvoyant à des notions dont les représentants sont jugés qualitativement peu différenciés, alors qu'il peut devenir obligatoire avec des termes désignant des entités fortement individuées, comme les animés humains ou les objets à contenu culturel ${ }^{3}$. Comme le souligne T. Nikolaeva (1985:45-46), c'est cette fonction individualisante qui les distingue des articles indéfinis des langues d'Europe occidentale, ces derniers marquant la simple appartenance d'une occurrence à une classe notionnelle, sans référence à ses propriétés singulières.

Si les nombreuses études consacrées aux adjectifs indéfinis décrivent en détail les conditions de leur concurrence, ainsi que les diverses valeurs dérivées (dépréciative, minorante, etc.) que chacun d'eux est susceptible de prendre en fonction des déterminations contextuelles, elles ignorent généralement le problème de leur place dans le syntagme nominal ${ }^{4}$. Bien qu'ils soient assez souvent postposés au substantif dans les exemples donnés pour illustrer telle ou telle valeur appréciative, cette inversion de l'ordre canonique n'est jamais commentée, les auteurs n'y voyant sans doute qu'une simple caractéristique de la langue orale familière.

Nos observations montrent cependant que ces variations positionnelles sont loin d'être aléatoires et ont une influence directe sur l'interprétation de l'adjectif indéfini. C'est cette influence que nous nous proposons d'étudier ici, notre hypothèse étant qu'elle n'est que la conséquence d'un mécanisme plus général que nous avons déjà eu l'occasion de mettre en évidence sur l'exemple des adjectifs possessifs et démonstratifs ${ }^{5}$. Nous pensons que quelle que soit la nature du déterminant épithète, son rejet après le substantif porteur de l'accent de syntagme n'est pas une simple variante stylistique, mais marque la dissociation des deux types d'opérations intervenant dans la construction de la valeur référentielle de tout syntagme nominal :

- constitution d'une entité nominale repérée par rapport à une situation réelle ou fictive Siti ;

\footnotetext{
${ }^{1}$ INaLCO (Paris) \& CNRS FRE 3326 : SeDyL/CELBS ; courriel : BonnotCh1 @ aol.com

${ }^{2}$ Par « occurrence », terme emprunté à la Théorie des Opérations Énonciatives de A. Culioli, nous désignons ici une entité prise dans une relation et ancrée situationnellement, qui « incarne » en la délimitant la notion dont elle vérifie les propriétés. Dans On entendit un chien aboyer, «un chien » construit une occurrence de la notion «être chien ».

${ }^{3}$ Cf. les contrastes : Я уже засыпал, как вдруг на улице затрещал (*какой-то) мотоцикл и разбудил весь дом. / ...как вдруг на улице заорал (какой-то) прохожий и разбудил весь дом. (Bonnot-Saoulski, 1983 : 22); Прежде чем поехать в Италию, Саше надо купить (*какой-нибудь) чемодан / ...Саче надо прочитать какую-нибудь (vs. *Ø) книгу по римской истории. (Kreisberg, 1986 : 252).

${ }^{4}$ Font exception deux auteurs qui, traitant de deux indéfinis différents, aboutissent à des conclusions en apparence opposées : V. Gurevič (1968), s’interrogeant sur la possible émergence d'articles en russe, considère que ètot et odin en position d'enclise perdent leur contenu sémantique et deviennent de simples outils grammaticaux équivalant respectivement aux articles défini et indéfini des langues occidentales ; D. Paillard (2001), étudiant le fonctionnement de vsjakij comparé au français tout, voit au contraire dans la postposition de l'indéfini une forme de «lexicalisation» («Postposé, vsjakij introduit une détermination qualitative du $\mathrm{N}$ comparable à celle qu'apporte un adjectif » (p. 287)). Il s'agit là d'effets locaux dont il est possible de rendre compte dans le cadre de l'hypothèse unitaire que nous proposons.

${ }^{5}$ Cf . Bonnot 2008, 2009, 2010 et (à paraître).
} 
- qualification de cette entité à travers la catégorisation opérée par le substantif et les spécifications exprimées par les déterminants.

De fait, l'absence devant le substantif de tout terme qui serait susceptible d'exprimer un repérage situationnel signifie que celui-ci n'est pas nécessaire : le référent est présenté comme déjà actualisé ; quant au déterminant rejeté en finale, perdant sa fonction d'ancrage, il n'exprime plus qu'une qualification sans lien avec la situation. En d'autres termes, le syntagme à séquence inversée ne construit pas une occurrence nouvelle, mais projette sur une occurrence déjà actualisée une représentation indépendante de la situation immédiate.

Ce mécanisme peut être représenté par le schéma suivant, où les parenthèses symbolisent le syntagme à déterminant postposé qui met en relation deux pôles au départ dissociés - une entité $[\mathrm{X}]_{\text {Siti }}$ déjà actualisée dans la situation Siti et une représentation $[\mathrm{Y}]_{\text {Dét }}$ constituée indépendamment de Siti :

$$
[\mathrm{X}]_{\text {Siti }} \rightarrow() \leftarrow[\mathrm{Y}]_{\text {Dét }}
$$

L'application de ce schéma à un syntagme de référence définie peut être illustrée par l'exemple suivant, tiré d'un corpus oral spontané :

\section{(В комнате включён телевизор)}

A. Это что (о телевизионной передаче)?

Б. Это четвёртая (программа)//

‥ А-А/ шпионский этот (фильм)// (Русская разговорная речь, Тексты: 256)

Comme on le voit, le syntagme «inversé » špionskij ètot identifie un référent $[\mathrm{X}]_{\text {Siti }}$ déjà actualisé, l'émission que l'interlocuteur est en train de regarder, avec une représentation [Y $]_{\text {Dét }}$ constituée hors de cette situation, celle d'un film d'espionnage dont le locuteur savait qu'il devait passer sur la quatrième chaîne. On remarque que ce mécanisme énonciatif modifie la sémantique du démonstratif ètot : il ne fonctionne plus comme un déictique pointant un objet proche de la situation immédiate, mais renvoie à un terme évoqué dans une situation antérieure, à l'instar de l'article défini par lequel on le traduira en français : «Ah! le film d'espionnage !»

Cette influence de l'ordre linéaire sur la sémantique des déterminants s'observe également dans le cas des adjectifs indéfinis, la postposition conduisant à marginaliser les propriétés singulières dont on a vu qu'ils marquaient l'existence. En effet, lorsque le référent est indéfini, la seule représentation indépendante de la situation que l'on peut projeter sur lui est une représentation générique : c'est celle qui découle des propriétés de la notion à laquelle renvoie le substantif et est $a$ priori associable à toute occurrence de celle-ci. En d'autres termes, alors que l'emploi d'un adjectif indéfini individualise le référent, sa postposition le désindividualise en l'assimilant à une occurrence quelconque de la notion qu'il incarne. Ce fonctionnement peut être illustré par l'exemple (2) :

(2) (Interrogatoire d'un jeune garçon qui pendant le tournage d'une émission de télévision sur une place de Moscou a brandi devant les caméras une pancarte défiant la milice)

- Зачем ты показывал плакат?

- Попросили...

- Кто тебя попросил поднять этот плакат? Кто тебе его дал? - повторил вопрос Корзун.

- Баба одна. Тётка в смысле...

- Кто она такая?

- А я знаю? Отпустите... Она денег дала, сказала, покажи плакат, а то мне неудобно, я болею, мне в толпу не пробраться. (А. Маринина, Седьмая жертва)

L'antéposition de l'indéfini serait peu souhaitable dans ce contexte où sont réalisées les deux conditions posées par notre schéma:

- le référent a déjà été actualisé par la désinence de pluriel de poprosili et par la question de l'interlocuteur. S'il n'avait pas déjà été introduit, l'indéfini devrait être antéposé. Cf. (2a) :

(2a) - Зачем ты показывал плакат?

- Понимаете, одна баба дала мне денег и сказала, покажи плакат, а то мне неудобно, я болею, мне в толпу не пробраться. 
- l'identification du référent est minimale et se réduit à une simple catégorisation l'incluant dans la classe des «bonnes femmes ». La mention sous forme d'un adjectif de toute propriété différentielle obligerait à antéposer l'indéfini :

(2b) - Кто тебя попросил поднять этот плакат? Кто тебе его дал?

- Одна странная баба (/Какая-то баба странная), я её не знаю.

Il convient cependant de noter que la postposition n'interdit pas qu'une telle précision soit donnée dans un second temps :

(2c) - Кто тебя попросил поднять этот плакат? Кто тебе его дал?

- Баба одна. Худая такая. Я её не знаю.

En effet, la postposition de l'indéfini ne signifie pas que le référent n'a pas de propriétés singulières, (le choix de l'adjectif odin signifie justement que le locuteur connaît certaines de ces propriétés), mais seulement qu'elles sont volontairement ignorées par le locuteur, ce qui dans un contexte d'interrogatoire tel que (2) s'interprète comme un manque d'empressement à répondre aux questions posées.

En résumé, la postposition des adjectifs indéfinis marque une opération de désindividuation à partir d'une individuation première, ce qui n'est pas équivalent à la non-individuation que marquerait l'absence de déterminant (l'emploi d'un indéfini est du reste obligatoire en (2), du fait du caractère hautement individué des référents animés humains).

Avant d'examiner la façon dont cette opération de désindividuation interfère avec la sémantique propre des différents adjectifs indéfinis, nous commencerons par examiner les différents modes de préactualisation du référent.

\section{Différents modes de préactualisation de $[\mathrm{X}]_{\mathrm{Siti}}$}

Nous avons vu que l'indéfini ne peut être postposé que si le référent du syntagme nominal a déjà été actualisé. Cette préactualisation peut prendre trois formes :

\section{1. $[\mathrm{X}]_{\text {Siti }}$ est préactualisé par le contexte verbal}

Le syntagme à séquence inversée est coréférent d'un terme déjà introduit dans le contexte gauche. La postposition se rencontre fréquemment en réponse à une question visant à identifier le référent (cf. (2)), ou dans les syntagmes en apposition. Cf. (3) :

(3) (Au moment d'acheter à sa femme des bottes élégantes, mais chères, un mari s'interroge sur ce que sera sa réaction.)

[...] Скажет, на кой они мне, такие дорогие! Лучше бы девчонкам чего-нибудь взял, пальтишечки какие-нибудь - зима подходит. (В. Шукшин, Сапожки)

La postposition est ici obligatoire, car elle seule permet de comprendre que le syntagme nominal ne fait que spécifier le référent du pronom čego-nibud'. Si celui-ci n'avait pas déjà permis de

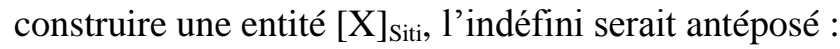

(3a) $[\ldots]$ Лучше бы какие-нибудь пальтишечки девчонкам взял - зима подходит.

On remarque par ailleurs que la relation de coréférence marquée par la postposition modifie l'interprétation de l'adjectif kakoj-nibud'. Alors qu'en (3a) les manteaux sont considérés pour euxmêmes, la variation exprimée par l'indéfini s'opérant au sein de la notion désignée par le substantif : «des manteaux dont les propriétés ne sont pas fixées », dans l'exemple d'origine (3), ils ne sont considérés que comme un exemple de ce que l'on doit acheter lorsque l'hiver approche et la variation qualitative s'étend cette fois à tout le champ sémantique regroupant les notions apparentées à «manteau »: «quelque chose comme des manteaux », «des manteaux ou tout autre vêtement d'hiver ». En l'absence de l'adjectif postposé, ce glissement sémantique entraîné par l'apposition devrait être explicité par un marqueur discursif tel que naprimer ou skažem :

(3b) [...] Лучше бы девчонкам чего-нибудь взял, пальтишечки, например (/скажем) - зима подходит. 


\section{2. $[\mathrm{X}]_{\text {Siti }}$ est préactualisé par la situation extralinguistique}

Le syntagme à séquence inversée a une valeur déictique et «pointe » un élément présent dans la situation.

Это была маленькая металлическая статуэтка - странный скорченный человечек в необычной позе. Он стоял на коленях, сильно наклонившись вперёд, упираясь тонкими руками в пьедестал. Меня поразило его лицо. С оскаленным кривоватым ртом, с тупым курносым носом, оно странно и дико глядело на нас выпуклыми белыми, видимо покрытыми эмалью, глазами. Лицо это было выполнено удивительно реалистично измученное, тоскливое, с упавшей на лоб жалкой прядью прямых волос. На голой спине человечка громадными буграми выдавались угловатые лопатки, колени были острые, а на руках торчали всего по три скрюченных когтистых пальца.

- Божок какой-то, - вполголоса сказал Строкулев. - Тяжелый. Золото, как ты думаешь?

Я поставил статуэтку на стол.

- Не похоже. Возможно, платина... (А. и Б. Стругацкие, Извне, l)

C'est la postposition de l'indéfini qui permet de comprendre que le syntagme nominal employé hors de toute construction syntaxique est destiné à identifier l'objet que les interlocuteurs ont sous les yeux. L'antéposition n'aurait été possible que si l'énoncé avait comporté un pronom déictique désignant explicitement cet objet :

(4a) - Похоже, это какой-то божок.

- Не думаю.

Pour autant, les deux variantes ne sont pas équivalentes. Avec la postposition, l'identité exacte du dieu représenté par la statuette n'intéresse pas le locuteur, qui est plutôt intrigué par le métal dans lequel elle est fondue («- Un petit dieu quelconque, murmura Stroguliov.»). Avec l'antéposition, l'impossibilité d'identifier ce dieu reste au premier plan et empêche le locuteur d'être trop affirmatif: il n'exprime qu'une hypothèse dont il convient d'examiner le bien-fondé, ce qui entraîne l'introduction d'un modalisateur et oblige à modifier le contexte de droite.

L'emploi déictique des syntagmes à déterminant indéfini postposé est particulièrement fréquent avec les substantifs appréciatifs exprimant une réaction du locuteur à un dire ou à une situation auxquels il est subitement confronté : Бред какой-то! (« C'est n'importe quoi !»), Прямо несчастье какое-то! («C'est vraiment pas de chance!»), Мистика какая-то! («C'est à n'y rien comprendre!»), etc.

\section{3. $[\mathrm{X}]_{\mathrm{Siti}}$ est préactualisé par son appartenance virtuelle à un ensemble déjà introduit}

Enfin, le référent peut n'avoir été préactualisé qu'indirectement, à travers la construction d'un ensemble auquel il est susceptible d'appartenir :

(Dialogue entre deux amis dont le second vient de perdre une personne très proche) - [...] Ты-то как сам?

- Хреново, брат! Очень хреново! Деда я хоронил, бабушек тоже. Приятель один хороший разбился на машине, давненько уже. А такого близкого и родного человека, молодого... терять ещё не приходилось. Да и не было у меня в сознательной жизни более близкого человека. [...] (Е. Гришковец, Асфальт)

L'antéposition de l'indéfini (Один хороший приятель разбился на машине) serait peu souhaitable dans ce contexte, car elle annoncerait un récit autonome sur le référent considéré dans sa singularité. Seule la postposition permet de souligner que ce référent n'est introduit que comme un élément de l'ensemble préconstruit par les deux propositions qui précèdent: celui des personnes proches que le locuteur a déjà vu mourir dans son entourage. C'est pourquoi ses propriétés individuelles importent peu, seules comptent ses caractéristiques notionnelles, qui l'opposent aux autres éléments de l'ensemble : la mort de grands-parents ou celle d'un ami du même âge constituent des expériences différentes. On remarque qu'ici la postposition de l'indéfini n'est pas bloquée par la présence de l'adjectif xorošij, lui aussi postposé, puisque celui-ci, contrairement à strannyj en (2b), 
nomme une propriété permettant de construire une sous-classe notionnelle contextuellement pertinente : celle des amis proches, dont la perte est plus douloureuse que celle des simples relations.

\section{Incidence de l'opération de désindividuation sur l'interprétation de l'adjectif indéfini}

Les valeurs produites par l'opération de désindividuation sont assez variées : elle dépendent à la fois du contexte et la sémantique propre de l'adjectif indéfini. Nous n'en donnerons ici que quelques exemples, en nous limitant aux trois indéfinis les plus courants : odin, kakoj-to et kakoj-nibud'.

\subsection{ODIN}

C'est l'indéfini qui exprime le degré d'indétermination le plus faible. Il signifie qu'un terme est distingué dans sa classe sur la base de propriétés singulières que le locuteur pourrait éventuellement expliciter, même s'il ne juge pas utile de le faire. En indiquant que le locuteur fait abstraction de ces propriétés singulières, la postposition met l'accent sur les propriétés définitoires de la notion désignée par le substantif, ce qui permet d'activer les représentations traditionnellement associées à celle-ci :

(6) (Le héros explique à son amie, jeune prostituée, qu'il est allé rendre à la femme de l'un de ses amis récemment décédé l'argent que celui-ci lui avait prêté.)

Лена понимающе кивнула.

- Мою подружку месяц назад убили. Пьяный один позвал к себе... И задушил. А я у неё книжки брала читать, у её предков классная библиотека... Но я назад не понесу... (А. Курков, Приятель покойника)

La postposition indique que le syntagme vient identifier le sujet déjà actualisé du procès ubili tout en conférant à cette identification une valeur explicative : le meurtrier est assimilé à un homme saoul prototypique, or chacun sait qu'un homme saoul n'est plus maitre de ses actes. Nos informateurs ont jugé que s'il avait été désigné par un substantif dont le sémantisme n'implique pas la prédisposition à des actes violents, l'antéposition aurait été plus naturelle :

(6а) - Мою подружку месяц назад убили. Один (богатый) клиент позвал её к себе... Напился и задушил.

\subsection{KAKOJ-TO/ČEJ-TO}

Les adjectifs indéfinis formés à l'aide du formant d'origine démonstrative -to expriment un degré d'indétermination plus élevé que odin et se chargent facilement de valeurs subjectives. Ils signifient qu'une occurrence est distinguée dans sa classe sur la base non de ses propriétés intrinsèques, mais de sa seule participation à une situation présentée comme réalisée (Paillard, 1984).

3.2.1. L'interprétation la plus courante est que les propriétés singulières de cette occurrence, bien que fixées par la situation, restent inconnues du locuteur. Dans les contextes de «discours » (au sens de Benveniste) marqués par une forte implication du locuteur, cette indication peut donner naissance à diverses valeurs subjectives ou modales dont la nature dépend en partie de la position de l'adjectif indéfini. Lorsqu'il est antéposé, ces propriétés ignorées sont présentées comme potentiellement pertinentes, d'où l'éventuelle émergence de valeurs comme l'incertitude (cf. (4a) ci-dessus) ou un sentiment d'inadéquation par rapport à la situation ${ }^{6}$; lorsqu'il est postposé, elles sont présentées comme sans intérêt, ce qui produit des valeurs d'un autre type, illustrées par les exemples (7) à (9) :

\section{Banalisation :}

(Découvrant un homme inconnu installé dans l'isba, une jeune paysanne interroge sa mère :)

- Мама, что это за человек у нас?

- Так... Проходящий какой-то. Странник, веру ищет, ночевать попросился.

(Ю. Казаков, Странник)

L'antéposition est exclue, car elle signifierait que la locutrice s'interroge elle-même sur l'identité exacte de l'homme, alors que, comme le montre la particule Tak, son but est au contraire de rassurer sa fille en soulignant qu'il ne possède aucune caractéristique particulière qui pourrait justifier une quelconque inquiétude de sa part («-Bah, c'est juste quelqu'un de passage »).

\footnotetext{
${ }^{6} \mathrm{Cf}$ : С какой-то грустью смотрел я на веселящуюся молодёж, mais : ' на умирающего больного. (Nikolaeva, 1985 : 55)
} 
(8) (Rentrant chez elle après une après-midi de courses, l'enquêtrice Nadia Kamenskaïa apprend par son mari qu'un de ses collègues la cherche partout.)

- Что случилось? - перепугалась она.

- А что у вас обычно случается? Труп чей-то обнаружили, - невозмутимо ответил Алексей.

- Чей труп?

- Не знаю, Асенька, твой друг мне не доложил. (А. Маринина, Реквием)

La postposition est rendue nécessaire par le ton pince-sans-rire du locuteur, qui typifie la situation en marquant ostensiblement ses distances par rapport au métier de sa femme. ( - Que veux-tu qu'il arrive dans votre métier? On a encore découvert un cadavre quelconque. ») L'antéposition aurait supposé une certaine implication personnelle de sa part (8a), alors que l'absence de déterminant aurait correspondu à une information totalement neutre $(8 \mathrm{~b})$ :

(8а) - Что случилось? - перепугалась она.

- В парке чей-то труп обнаружили. Придётся ехать.

(8b) (Attroupement dans la rue)

- Что случилось?

- Труп обнаружили.

Mépris :

(9) (Une jeune fille essaie d'être reçue par un chanteur célèbre dont la porte est gardée par un cerbère aux larges épaules.)

- Скажите ему, что пришла дочь композитора Немчинова.

Детина хмыкнул, но глаза перестали казаться пустыми, в них мелькнуло что-то вроде любопытства. Будто нехотя снял он трубку и набрал номер.

- Вячеслав Олегович? Это дежурный. Тут к Игорю девочка пришла, говорит, что она дочь композитора какого-то...

- Немчинова, - тут же подсказала Лера. - Геннадия Немчинова. (А. Маринина, Реквием)

En refusant de répéter le nom du compositeur et en ramenant celui-ci à un représentant quelconque de sa classe, le locuteur continue d'afficher envers la jeune fille le mépris qu'il avait déjà exprimé par ses mimiques (cf . xmyknul, budto nexotja) : «...elle prétend qu'elle est la fille de je ne sais quel compositeur... $\gg^{7}$. L'antéposition aurait correspondu à une attitude plus neutre, où il se serait contenté de transmettre une information en soulignant qu'il n'en connaissait pas tous les détails, ce qui aurait été plus naturel dans une situation où il aurait effectivement ignoré le nom du référent. Cf. (9a) :

- Скажите ему, что пришла дочь члена Союза композиторов.

- Вячеслав Олегович? Это дежурный. Тут к Игорю девочка пришла, говорит, что она дочь какого-то композитора... (« ... elle dit qu'elle est la fille d'un compositeur. »)

3.2.2. La valeur dépréciative illustrée par (9) peut également apparaître lorsque kakoj-to est antéposé, mais il faut pour cela que le contexte soit tel que l'interlocuteur ne puisse interpréter l'indéfini comme une marque d'ignorance. La valeur dépréciative naît alors de la contradiction entre les déterminations contextuelles et la sémantique de l'indéfini : on comprend que si le locuteur ne distingue pas le référent sur la base de ses propriétés singulières, ce n'est pas parce qu'il les ignore, mais parce qu'il considère qu'il n'en vaut pas la peine. Un tel glissement sémantique s'observe par exemple en (10), où le syntagme indéfini est employé de façon prédicative pour qualifier un référent déjà identifié :

\footnotetext{
${ }^{7}$ En (9), la préactualisation du référent est opérée lexicalement, par l'emploi du mot relationnel doč', dont $[\mathrm{X}]_{\mathrm{Siti}}$ est une valence obligatoire.
} 

так, как будто он старшина рота. (А. Зиновьев, Светлое будущее)

Même dans un contexte de ce type, où toute idée d'indétermination disparaît au profit de la seule valeur appréciative, antéposition et postposition ne sont pas équivalentes, comme le montre la comparaison avec (11), qui pourrait sembler très proche :

(11) (Une inspectrice de la police criminelle enquête sur une affaire survenue lors du tournage d'un film: la femme du scénariste a disparu et le chauffeur de l'équipe a été assassiné. Il s'avère qu'il était mêlé à de petits trafics, mais l'inspectrice comprend que ce n'est pas cette piste que souhaite privilégier son chef, l'ambitieux Afanassiev, surnommé Afonia)

[...] По большому счёту это направление работы интересует Афоню меньше всего. Подумаешь - водитель какой-то, из убийства водителя имени себе не сделаешь. А вот убийство и похищение с целью срыва съёмок - это может прозвучать, во всех газетах напишут. [...] (А. Маринина, Незапертая дверь)

La différence est que l'antéposition exprime un jugement émis par rapport à la situation, en réaction à la façon dont le référent se manifeste dans celle-ci, alors que la postposition exprime, conformément à notre hypothèse, un jugement indépendant de la situation et portant sur le référent considéré pour lui-même. C'est pourquoi nos informateurs ont jugé que seule l'antéposition était possible en (10), où le locuteur n'exprime pas d'hostilité particulière pour son ancien étudiant, mais s'irrite seulement de voir celui-ci le tutoyer, alors que la postposition est préférée en (11), où c'est de par sa profession même que le chauffeur fait figure de quantité négligeable aux yeux des journalistes et du chef de la police criminelle.

Le fait que l'indéfini postposé exprime un jugement sur le référent considéré pour lui-même explique pourquoi seule l'antéposition semble possible pour exprimer une appréciation quantitative :

(12) З За какой-то час он выкопал большую яму. (exemple emprunté à Kuz'mina, 1989 :166)

En effet, une durée n'est pas longue ou brève en soi, mais uniquement par rapport à ce qu'elle a permis de réaliser.

\subsection{KAKOJ-NIBUD'}

Conformément à l'étymologie de -nibud', cet indéfini non spécifique signifie que les propriétés du référent sont susceptibles de varier, ce qui est le plus souvent dû au fait que sa sélection au sein de sa classe dépend de la réalisation d'une situation encore hypothétique. La place de l'indéfini dépend de l'attitude exprimée par le locuteur par rapport à cette variabilité : avec l'antéposition, ces propriétés encore non fixées ne sont a priori pas toutes équivalentes pour lui ${ }^{8}$, alors qu'avec la postposition, elles sont présentées comme interchangeables. Cette indifférence affichée par rapport à l'indétermination exprimée par l'adjectif peut donner naissance à des valeurs subjectives de dédain ou de distanciation proches de celles qui ont été observées avec kakoj-to postposé :

(13) (Un gaillard à la stature impressionnante passe commande au restaurant)

- Два шашлыка, пару салатов каких-нибудь и курицу в табаке.

- Табака, - поправила официантка, записывая.

- Я знаю, - сказал детина. - Я же шучу. (В. Шукшин, Случай в ресторане)

Dans une situation actualisant un ensemble de plats entre lesquels le locuteur est censé opérer un choix, l'indication explicite qu'il ne fait aucune différence entre les salades proposées fait comprendre que pour lui, elles ne constituent pas de la vraie nourriture, mais sont de simples « mises en bouche » tout juste bonnes à faire passer le temps en attendant la viande. L'expression de la moindre préférence quant à leur composition entraînerait le retour à l'antéposition :

(13a) - Два шашлыка и пару каких-нибудь салатов, только без огурцов, если можно.

\footnotetext{
${ }^{8}$ La glose «bezrazlično kakoj» proposée par S. Kuz'mina (1989: 175) semble peu adéquate, d'autant qu'elle écrit plus loin : «V bol'šinstve kontekstov značenie bezrasličnosti ne voznikaet ili ne javljaetsja očevidnym.»
} 
Dans d'autres cas, comme on l'a vu en (3) (pal'tišečki kakie-nibud'), la postposition peut signifier que la variation qualitative ne reste pas limitée à la notion désignée par le substantif, mais s'étend à un ensemble de notions apparentées. Cf. (14) :

- Налей мне чайку какого-нибудь холодненького, что-то всё во рту... язык как деревянный. (Г. Семёнов) ${ }^{9}$

Ce glissement sémantique nous semble naître de la mention dans le contexte d'une propriété que doit vérifier le référent (« être utile pour l'hiver » en (3), « être bien froid » en (14)), alors même que la postposition de l'indéfini présente sa variation qualitative comme indifférente. On comprend que pourvu qu'il vérifie cette unique propriété, toutes les autres importent peu, y compris celles dont dépend son rattachement effectif à la notion désignée par le substantif.

Avec l'antéposition, le même glissement sémantique n'apparaît que si l'indéfini détermine un nom à référent unique (nom propre) ou renvoyant à une notion au sein de laquelle toute variation est exclue. (- Nalej mne kakogo-nibud' čajku exprimerait une possibilité de choix entre plusieurs variétés de thé.)

\section{Conclusion}

La postposition des adjectifs indéfinis interfère avec leur fonction individualisante en indiquant que les propriétés singulières auxquelles ils renvoient ne sont pas pertinentes pour la situation où le référent est actualisé. Les effets de sens produits, qui dépendent à la fois du contexte et de la nature de l'indéfini, vont de la simple assimilation du référent à une occurrence prototypique de la notion qu'il incarne à diverses valeurs appréciatives et modales telles que indifférence, mépris ou ironie. Ces valeurs faisant intervenir la subjectivité de l'énonciateur, leur émergence est caractéristique des contextes de «discours ». Il resterait à étudier le cas des contextes narratifs, où la postposition des indéfinis, tout comme celle des possessifs et des démonstratifs (Bonnot, à paraître), est la marque d'un dédoublement de l'instance narrative (discours indirect libre, construction d'une instance d'observateur, etc.).

\section{RÉFÉRENCES BIBLIOGRAPHIQUES}

Bonnot-Saoulski Ch., 1983, «L'étude des indéfinis dans une théorie de l'énonciation » // III Colloque de Linguistique russe, Aix-en-Provence, 15-17 mai 1981, Paris : Institut d'Etudes Slaves, 11-24.

Bonnot, Ch., 2008, «Un cas d' « inversion » de l'ordre canonique en russe moderne : la postposition du pronom possessif épithète » // Mélanges offerts au Professeur M. Guiraud-Weber, Aix-en-Provence : Publications de l'Université de Provence, 39-54.

2009, « Du syntagme au texte. A propos d'une variation de l'ordre des mots dans le syntagme nominal en russe moderne " // La cohérence du discours dans les langues slaves. Linguistique théorique et textuelle, Paris : Revue des études slaves, LXXX/1-2, 161-173.

2010, «Identification et préconstruit : à propos d'une variation de l'ordre linéaire dans le syntagme nominal russe » // Construction d'identité et processus d'identification, Bern-Berlin-Bruxelles-Frankfurt/Main-New York-OxfordWien : Peter Lang, 15-32.

(à paraître), «Postposition des déterminants déictiques et dédoublement du narrateur. (Sur l'exemple des récits à la première personne) », Narration et énonciation, Slovo, Paris : Publications Langues 'O.

Culioli, A., 1999a, «Structuration d'une notion et typologie lexicale. A propos de la distinction dense, discret, compact » // Pour une linguistique de l'énonciation, Domaine notionnel, t. 3, Gap-Paris : Ophrys, 9-15. $1999 \mathrm{~b}$, « A propos de quelque » // ibidem : 49-58.

Gurevič V. V., 1968, « Est' li artikli v russkom jazyke ? », Russkaja reč', 1968/3, Moscou : Nauka, 57-59.

Kreisberg A., 1986, «Certains cas d'emploi obligatoire des adjectifs indéfinis en russe » // IV Colloque de linguistique russe, Toulouse, 18, 19 et 20 mai 1984, Paris : Institut d'Etudes Slaves, 247-255.

Kuz'mina, S. M., 1989, «Semantika i stilistika neopredelënnyx mestoimenij» // Grammatičeskie issledovanija funkcional'nostilističeskij aspekt. Supersegmentnaja fonetika, Morfologičesja semantika, Moscou : Nauka, 158-231.

Nikolaeva T. M., 1985, Funkcii častic v vyskazyvanii (na materiale slavjanskix jazykov), Moscou : Nauka.

Padučeva E. V., 1985, Vyskazyvanie i ego sootnesënnost's dejstvitel'nost'ju, Moscou : Nauka.

Paillard D., 1984, Enonciation et détermination en russe contemporain, Paris : Institut d'Etudes Slaves.

Paillard D., 2001, «Tout $N$ en français versus vsjakij $N$ en russe », Détermination et Formalisation, X. Blanco, P.-A. Buvet et Z. Gavriilidou (eds.), Amsterdam/Philadelphia : John Benjamins, 273-290.

Russkaja razgovornaja reč', Teksty, 1978, E. A. Zemskaja et L. A. Kanapadze (eds.), Moscou : Nauka.

Selivërstova O. H., 2004, Mestoimenija v jazyke i reči // Trudy po semantike, Moscou : Jazyki slavjanskoj kul'tury, 2004 : $375-556$.

\footnotetext{
${ }^{9}$ exemple emprunté à Kuz’mina (1989 : 177) qui glose : «pit’ja vrode čaja » sans commenter la postposition.
} 
Thach J. D., 2009, «na:/ ?cj: Indéfini - Interrogatif en khmer. Deux formes de non-individuation », Faits de Langues - Les Cahiers, 1, 119-159.

Veyrenc J., 1994, « Les indéfinis en russe » // L’indéfini, Faits de langues 4/1994, Paris : PUF, 105-112. 